\title{
Lasing of ZnO Micro / Nanocrystals by Ultraviolet Laser Excitation and Atmospheric Alignment with Laser-induced Motions and Electrical Field
}

\author{
Kota OKAZAKI, Tetsuya SHIMOGAKI, Daisuke NAKAMURA, Mitsuhiro HIGASHIHATA and Tatsuo OKADA \\ Graduate School of Information Science and Electrical Engineering, Kyushu University, \\ 744, Motooka, Nishi-ku, Fukuoka, 819-0395, Japan \\ E-mail: okazaki@laserlab.ees.kyushu-u.ac.jp
}

\begin{abstract}
Lasing characteristics and an alignment method of $\mathrm{ZnO}$ micro / nanocrystals were investigated for an application to ultraviolet (UV) laser diode (LD). A single $\mathrm{ZnO}$ microsheet synthesized by carbothermal chemical vapor deposition method was examined by spectra observation with a thirdharmonic Nd:YAG laser $(\lambda=355 \mathrm{~nm}, \tau=5 \mathrm{~ns})$ as an excitation laser light. The observed emission spectra showed the lasing characteristics having mode structure and a threshold for lasing at 49 $\mathrm{kW} / \mathrm{cm}^{2}$. In addition, laser-induced motions (LIM) of $\mathrm{ZnO}$ micro / nanocrystals at high excitation intensity beyond the lasing threshold were observed when those were excited by the UV laser beam at a repetition rate of $20 \mathrm{~Hz}$. The mechanism of the LIM was discussed, and an alignment method of $\mathrm{ZnO}$ micro / nanocrystals using the LIM and electrical fields was demonstrated.
\end{abstract}

DOI:10.2961/jlmn.2012.03.0006

Keywords: $\mathrm{ZnO}$ micro / nanocrystals, ultraviolet (UV), lasing, laser-induced motions (LIM), electrical field, atmospheric alignment

\section{Introduction}

Zinc oxide $(\mathrm{ZnO})$ is a II-VI semiconductor material with a wide band-gap energy of $3.37 \mathrm{eV}$ and has similar characteristics to Gallium Nitride $(\mathrm{GaN})$ which has been already used in ultraviolet (UV) light emission devices. However, $\mathrm{ZnO}$ has several advantages over GaN, including abundant natural resources of $\mathrm{Zn}$ compared to scarce resources of $\mathrm{Ga}$ and a considerably larger exciton binding energy of $\mathrm{ZnO}(60 \mathrm{meV})$ than that of $\mathrm{GaN}(28 \mathrm{meV})$ and the thermal energy at room temperature $(26 \mathrm{meV})$. Therefore, $\mathrm{ZnO}$ can be a promising material for $\mathrm{UV}$ device applications.

$\mathrm{ZnO}$ micro / nanocrystal has an excellent crystallinity due to its small volume, and UV stimulated emission from various $\mathrm{ZnO}$ nanocrystals has been reported ${ }^{[1,2]}$. Those reports show that $\mathrm{ZnO}$ nanocrystals can be a candidate for an excellent UV laser medium. In particular, a number of studies on lasing from $\mathrm{ZnO}$ nanowire due to a microcavity effect have been reported ${ }^{[3,4]}$. However, we have reported comparison of lasing characteristics from both a single $\mathrm{ZnO}$ nanosheet and a single $\mathrm{ZnO}$ nanowire by $\mathrm{UV}$ laser beam excitation, and it was considered that the $\mathrm{ZnO}$ nanosheet could be superior laser medium due to its lower lasing threshold than the threshold of the $\mathrm{ZnO}$ nanowire ${ }^{[5}$,

6]. In that report, lasing mechanism on the single $\mathrm{ZnO}$ nanosheet was also discussed and concluded that the lasing was attributed to the microcavity effect due to the UV light confinement within the nanosheet ${ }^{[5]}$.

$\mathrm{ZnO}$ micro / nanocrystal can be an excellent UV laser medium and an attractive UV laser light source with its quite-small dimension of several hundred $\mathrm{nm}$ in the minimum diameter. Furthermore, a simple and practical fabrication method of a UV laser diode without complicated processes such as a lithography method can also be expected by an alignment of $\mathrm{ZnO}$ crystals playing a role of building blocks as UV laser mediums. However, there is a difficulty on the handling those $\mathrm{ZnO}$ micro / nanocrystals because of its small dimension. Even though an alignment method using a dielectrophoretic process have been reported, a dielectric solution imposing restrictions on other electrical devices is required ${ }^{[7,8]}$.

In this study, we investigate lasing characteristics of a single $\mathrm{ZnO}$ microsheet excited by a pulsed UV laser beam. In addition, laser-induced motions (LIM) of $\mathrm{ZnO}$ micro / nanocrystals dispersed on a substrate during the UV laser irradiation at a relatively high excitation power density beyond the lasing threshold is also observed, and the atmospheric alignment of $\mathrm{ZnO}$ crystals by the combination of the LIM and an electrical field will also be suggested.

2. Synthesis of $\mathrm{ZnO}$ Micro / Nanocrystals and Setup for Lasing Characteristics Measurement

$\mathrm{ZnO}$ micro / nanocrystals were synthesized by a carbothermal chemical vapor deposition (CVD) method. A silicon substrate was employed for the deposition of heated $\mathrm{ZnO}$ vapor. In advance, a gold thin film with a thickness of $1 \mathrm{~nm}$ was evaporated onto silicon substrate, which was performed by a vacuum deposition equipment. The evaporated gold serves as a catalyst promoting the synthesis of $\mathrm{ZnO}$ crystals on the substrate. The mixed source of $\mathrm{ZnO}$ powder and graphite which is an evaporation promoter was placed in an alumina boat, and the silicon substrate was located above about $10 \mathrm{~mm}$ from the $\mathrm{ZnO}$ source where the gold-evaporated surface was opposed to the source. The prepared alumina boat was inserted to a silica glass vacuum chamber, and the air in the camber was evacuated up to several Torr by a vacuum pump. Then the mixed gases of argon (Ar) and oxygen $\left(\mathrm{O}_{2}\right)$ were flowed at the amount of $100 \mathrm{sccm}$ of Ar and $3 \mathrm{sccm}$ of $\mathrm{O}_{2} . \mathrm{ZnO}$ micro / nano wires and sheets were synthesized at the temperature of $1100{ }^{\circ} \mathrm{C}$ and the ambient gas pressure of 300 Torr during $30 \mathrm{~min}$. 

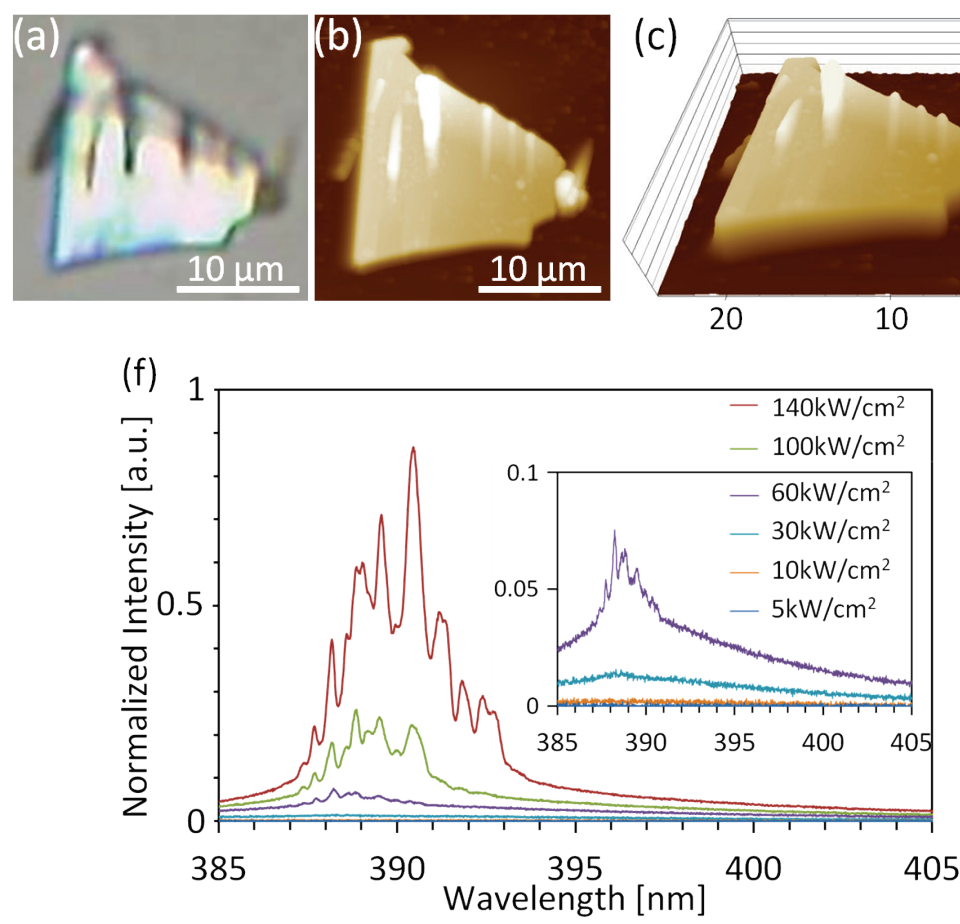

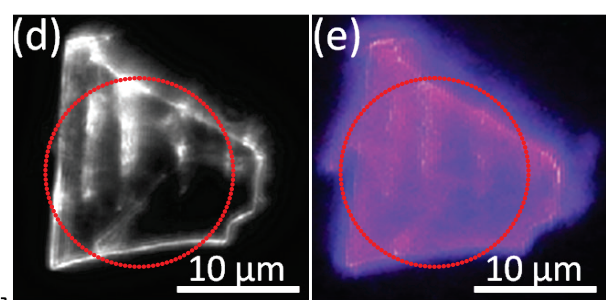

$0[\mu \mathrm{m}]$

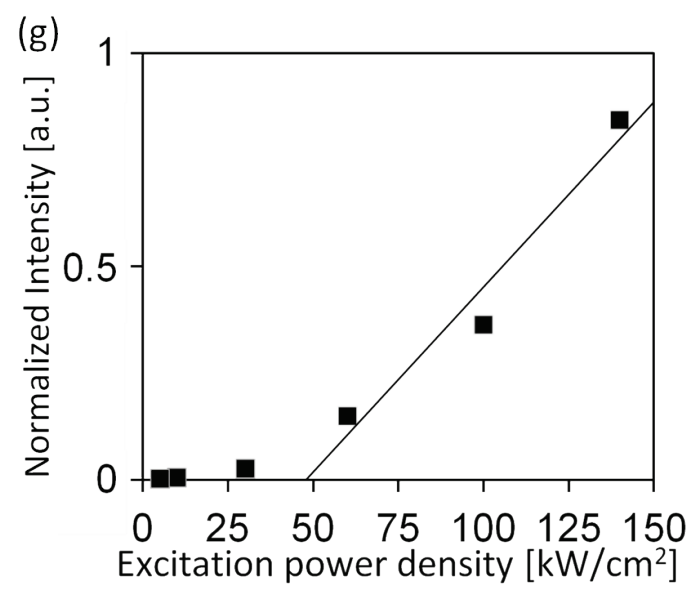

Fig. 1 (a) Typical color CCD image of a single $\mathrm{ZnO}$ microsheet lying on a silica glass substrate. (b) AFM image of the $\mathrm{ZnO}$ microsheet. (c) 3D image of the AFM result. The thickness of the microsheet was estimated to be about $1.5 \mu \mathrm{m}$. (d) Monochrome CCD image and (e) typical color CCD image of the microsheet under a light excitation with third harmonic of a Q-switched Nd:YAG laser ( $\lambda=355 \mathrm{~nm}, \tau=5 \mathrm{~ns}$ ). (d) Emission spectra from the area encircled with red-broken line in Fig. 1 (d) and (e) in changing the excitation power densities. (e) Input-output characteristics of the ZnO microsheet where every peak at $\lambda=388.2 \mathrm{~nm}$ in Fig. 1 (f) was plotted against the excitation power densities.

For the investigation of lasing characteristics, emission spectra from a $\mathrm{ZnO}$ micro / nanocrystal excited by third harmonic of a Q-switched Nd:YAG laser beam $(\lambda=355$ $\mathrm{nm}, \tau=5 \mathrm{~ns}$ ) (Polaris II, NEW WAVE) was observed by a spectrometer (SA-100S-HPCB/TTK, Lambda Vision). The $\mathrm{ZnO}$ crystals synthesized on the silicon substrate were dispersed on a silica glass substrate by an ultrasonic cleaner method with ethanol solution. The excitation laser beam with a diameter of approximately $5 \mathrm{~mm}$ was injected through the silica glass substrate without any focusing, and the emission light from an excited $\mathrm{ZnO}$ crystal was acquired by an objective lens with a magnification factor of 100 and transferred to the spectrometer with a silica glass fiber. In this observation setup, the area observed by the spectrometer was approximately $15 \mu \mathrm{m}$ in diameter due to the employment of the objective lens, and therefore, emission light from a single $\mathrm{ZnO}$ micro / nanocrystal could be observed. $\mathrm{ZnO}$ crystals lying on a silica glass substrate was observed and positioned by an integrated monochrome charge-coupled device (CCD) (DS-Qi1 Mc, Nikon).

\section{Lasing Characteristics of $\mathrm{ZnO}$ Microsheet}

Figure 1 (a) shows a typical optical microscope image of a single $\mathrm{ZnO}$ microsheet on a silica glass substrate. The microsheet was observed by an atomic force microscope (AFM) (KEYENCE, VN-8000M/8010M) as shown in Fig. 1 (b) and the thickness was estimated to be about $1.5 \mu \mathrm{m}$ from the 3D image in Fig. 1 (c). From those images, the microsheet has several bone-like structures in a longitudinal direction, which is attributed to the synthesis mechanism of $\mathrm{ZnO}$ micro / nanosheets by the CVD method. First, a 1D basal $\mathrm{ZnO}$ wire is synthesized, and parallelsidebranch $\mathrm{ZnO}$ wires perpendicularly grow on the basal wire. Finally, a $\mathrm{ZnO}$ sheet is formed by filling those interspaces between sidebranch $\mathrm{ZnO}$ wires ${ }^{[9]}$.

Lasing characteristics on the prepared $\mathrm{ZnO}$ microsheet were investigated. The monochrome CCD image and a typical color $\mathrm{CCD}$ image of the $\mathrm{ZnO}$ microsheet under the light excitation was shown in Fig. 1 (d) and (e) where emission light from the encircled area with red-broken line was observed by the spectrometer. Figure 1 (f) shows the emission spectra from the microsheet when the excitation power densities were changed. The emission intensities increased with the increase of the excitation power densities, and the mode structures between narrow emissions from $387 \mathrm{~nm}$ to $393 \mathrm{~nm}$ due to the onset of lasing could be observed on broad emissions due to a nearband-edge emission. Even though every emission spectrum was acquired by the accumulated number of 600 times, every peak of mode structure appeared at the specific wavelength regardless of the different excitation power densities. It indicates that laser oscillation would take place within the microsheet by the confinement of UV light due to the high refractive index of $\mathrm{ZnO}$ of about 2.4 for the wavelength around $389 \mathrm{~nm}{ }^{[10]}$. The detailed investigations have been discussed in the earlier report ${ }^{[5]}$. Figure $1(\mathrm{~g})$ shows the input-output characteristics, and the lasing threshold of the $\mathrm{ZnO}$ microsheet was estimated to be about $49 \mathrm{~kW} / \mathrm{cm}^{2}$ which was considerably low as well as the $\mathrm{ZnO}$ nanosheet in the above reports ${ }^{[5,6]}$. 

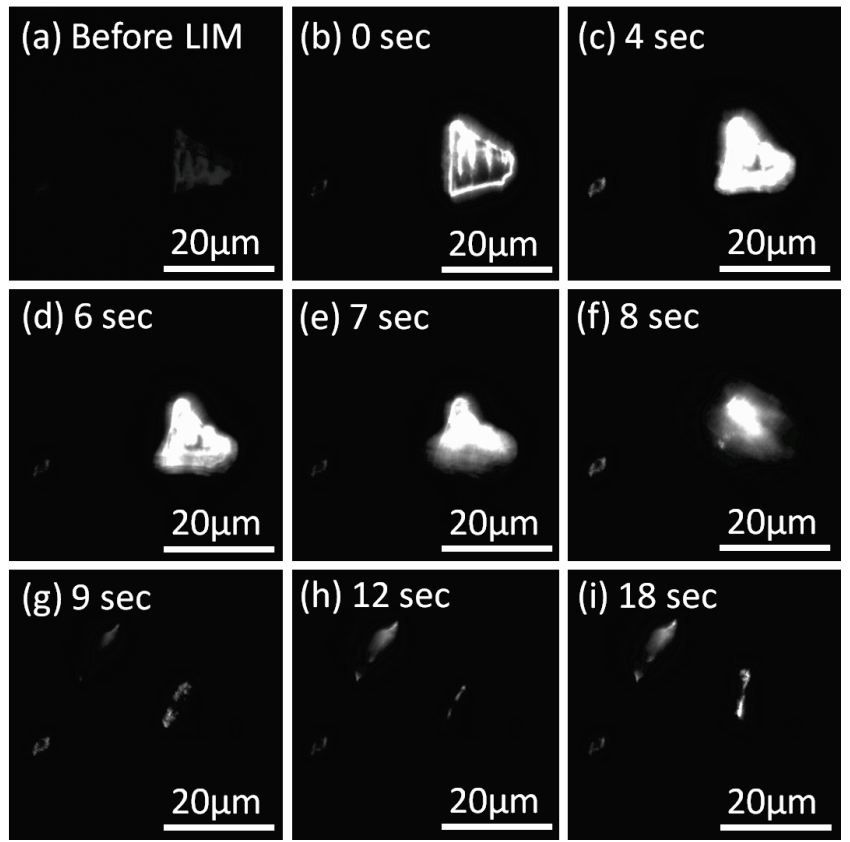

Fig. 2 Laser-induced motion of the same $\mathrm{ZnO}$ microsheet in Fig. 1. The sheet was irradiated by third harmonic of a Q-switched Nd:YAG laser $(\lambda=355 \mathrm{~nm}, \tau$ $=5 \mathrm{~ns}$ ) at an excitation power density of $550 \mathrm{~kW} / \mathrm{cm}^{2}$ and a repetition rate of $20 \mathrm{~Hz}$. Those images were acquired at a frame rate of 100 fps. The sheet was lifted up on a silica glass substrate by the laser irradiation.

\section{Laser-induced Motions of ZnO Micro / Nanocrystals}

At a large excitation power density around several hundred $\mathrm{kW} / \mathrm{cm}^{2}$ completely beyond the lasing threshold, laser-induced motions (LIM) of $\mathrm{ZnO}$ micro / nanocrystals irradiated by the UV laser beam without focusing were observed in the atmospheric condition. Figure 2 shows an example of the LIM of the $\mathrm{ZnO}$ microsheet which is the same sample as that in Fig. 1. When the microsheet was excited at $550 \mathrm{~kW} / \mathrm{cm}^{2}$ and a repetition rate of $20 \mathrm{~Hz}$, the microsheet was gradually lifted up on the silica glass substrate. Then, the microsheet was blown off as shown in Fig. 2 (f) and stood up on the substrate. Figure 3 shows the microsheet standing on the substrate after the LIM. Figure 3 (a1) and 3 (a2) shows typical color images focused on the bottom and the top of the standing microsheet, respectively. Similarly, monochrome images focused on the bottom and the top during the excitation at $190 \mathrm{~kW} / \mathrm{cm}^{2}$ were shown in Fig. 3 (b1) and 3 (b2), respectively. Interestingly, the microsheet keep standing in stable condition, and the emission light spectra from the standing microsheet was observed as shown in Fig. 3 (c) where the encircled area
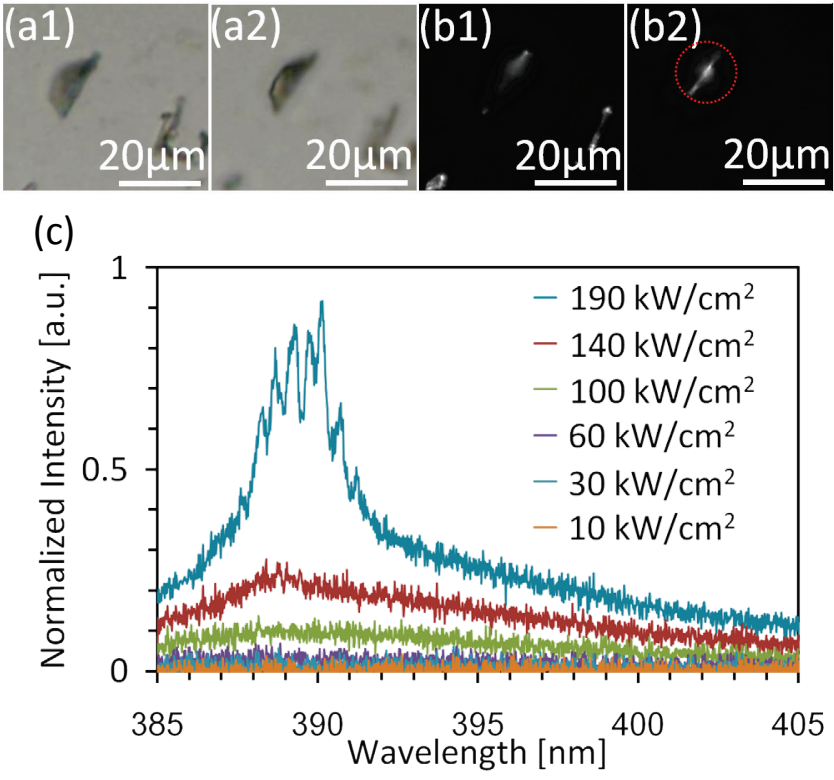

Fig. $3 \mathrm{CCD}$ images of the upright $\mathrm{ZnO}$ microsheet after the laser-induced motion. The microsheet is the same as that in Fig. 1 and Fig. 2. Color CCD images focused on (a1) the bottom and (a2) the top of the microsheet and monochrome CCD images of (b1) the bottom and (b2) the top during the excitation at $190 \mathrm{~kW} / \mathrm{cm}^{2}$ were shown. (c) Emission spectra from the area encircled by red-broken line in Fig. 3 (b2).

with red-broken line in Fig. 3 (b2) was the observation area. From the observed spectra, the microsheet still remains the lasing characteristics after the LIM. The reason why the micro / nanosheet stood in stable condition on the substrate might be attributed to an electrostatic force and an atomic force because those adhesion forces were considerably larger than the weight of a micro / nanocrystal ${ }^{[11]}$.

As well as the $\mathrm{ZnO}$ microsheet, LIM of a $\mathrm{ZnO}$ nanowire was also observed. Figure 4 shows the random LIM like a sliding motion of a $\mathrm{ZnO}$ nanowire on a silica glass substrate. The nanowire was excited at $450 \mathrm{~kW} / \mathrm{cm}^{2}$ which was beyond the lasing threshold, and the amplified UV light was emitted from the both of edges of the nanowire because the nanowire plays a role of a waveguide. The diffraction pattern from the emission light could be observed, which is similar to the reported phenomenon ${ }^{[3]}$.

For further consideration of LIM, Al thin film with a thickness of several hundred $\mathrm{nm}$ on a silica glass substrate was prepared by a vacuum evaporation system. When the Al thin film was irradiated by the UV laser beam, a part of the $\mathrm{Al}$ thin film was stripped from the substrate and the
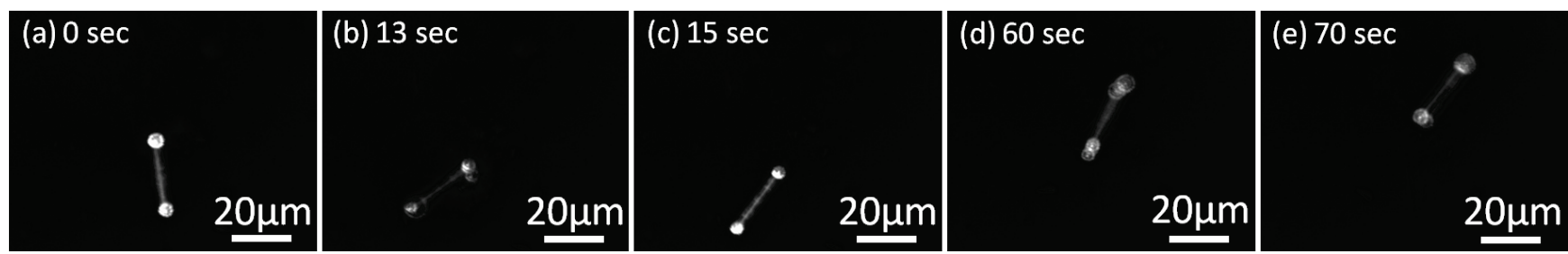

Fig. 4 (a) Time-course images of a random laser-induced motion of a $\mathrm{ZnO}$ nanowire lying on silica glass substrate. The nanowire was irradiated by third harmonic of a Q-switched Nd:YAG laser ( $\lambda=355 \mathrm{~nm}, \tau=5 \mathrm{~ns})$ at $450 \mathrm{~kW} / \mathrm{cm}^{2}$ and a repetition rate of $20 \mathrm{~Hz}$. Those images were acquired at a frame rate of $100 \mathrm{fps}$. 

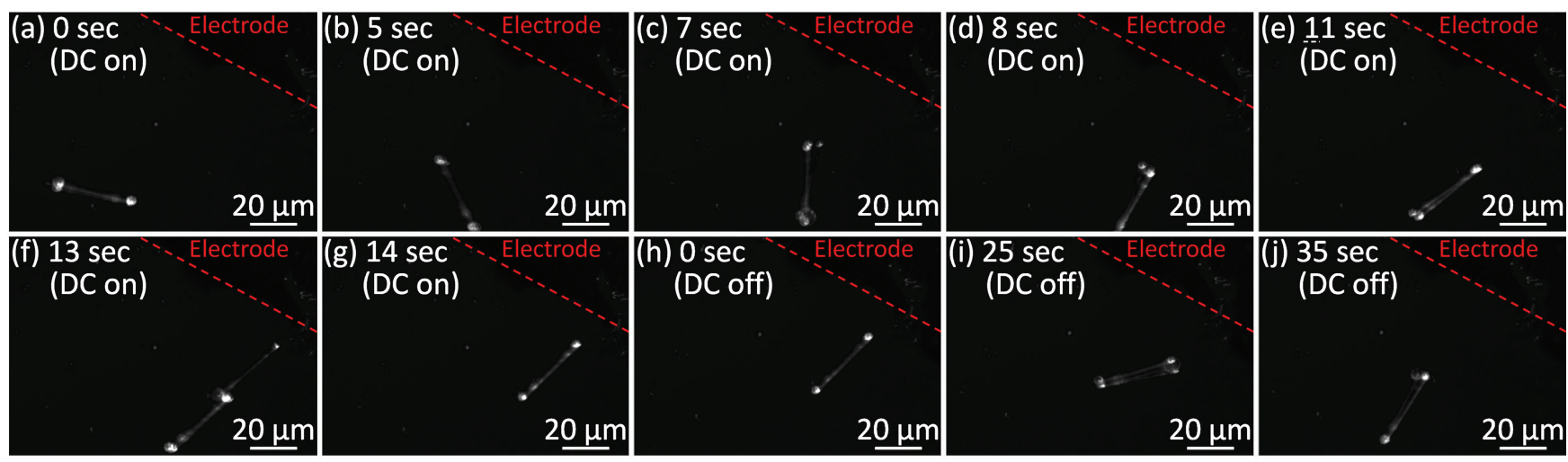

Fig. 5 Time-course images of a laser-induced motion controlled by electrical field applied by a DC power supply between triangular Al electrodes on a silica glass substrate. The schematic was shown on the right side. Those images were acquired at a frame rate of $100 \mathrm{fps}$. The $\mathrm{ZnO}$ nanowire was moved by third harmonic of a Q-switched Nd:YAG laser beam $(\lambda=355 \mathrm{~nm}, \tau=5 \mathrm{~ns})$ at $500 \mathrm{~kW} / \mathrm{cm}^{2}$ and a repetition rate of $20 \mathrm{~Hz}$. $(\mathrm{a} \sim \mathrm{f})$ The nanowire was moved and aligned along the electrical field and $(\mathrm{g})$ stopped at a stable position where the electrical field intensity was approximately $100 \mathrm{kV} / \mathrm{m}$. $(\mathrm{h} \sim \mathrm{j})$ The nanowire was randomly moved after DC power was turned off.

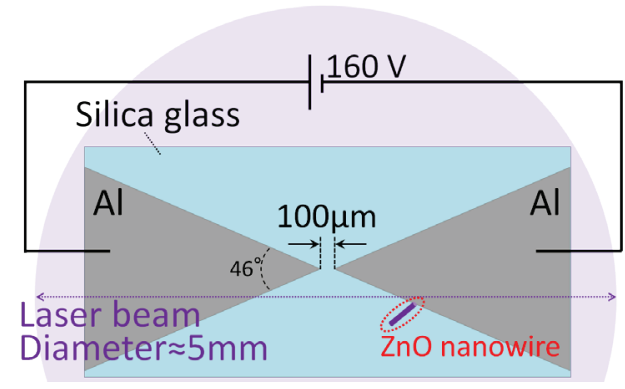

same LIM was observed as well as the $\mathrm{ZnO}$ crystals. Though LIM due to a fractional ablation of a $\mathrm{ZnO}$ crystal can be considered, the excitation at $450 \mathrm{~kW} / \mathrm{cm}^{2}$ corresponding to $2.3 \mathrm{~mJ} / \mathrm{cm}^{2}$ would be too small to ablate a $\mathrm{ZnO}$ crystal. The typical laser annealing of a $\mathrm{ZnO}$ crystal with a nanosecond pulsed UV laser which induces surface melting and photoluminescence improvement in UV emission is effective over several tens to a hundred $\mathrm{mJ} / \mathrm{cm}^{2}$ $[12,13]$. Therefore, the LIM would be caused by a strong acceleration due to a rapid thermal expansion of $\mathrm{ZnO}$ micro / nanocrystal. As a similar example, removal of micro / nanoparticle contaminations on a substrate can be carried out by laser cleaning method with a pulsed UV laser ${ }^{[11]}$. If micro / nanoparticles have a high absorption coefficient for the UV light, rapid thermal expansion is caused during the short laser pulse duration, resulting in the generation of strong acceleration. $\mathrm{ZnO}$ has also high absorption coefficient $\left(2.9 \times 10^{5} \mathrm{~cm}^{-1}\right)$ at $\lambda=355 \mathrm{~nm}^{[14]}$.

When a $\mathrm{ZnO}$ crystal is irradiated by a UV laser at fluence $F$ and pulse duration $\tau$, the absorbed light was converted to the emission light within the $\mathrm{ZnO}$ crystal at the internal quantum efficiency $\eta$. Therefore, the temperature rise $\Delta T$ of the surface is expressed as $\Delta T=(1-$ $R)(1-\eta) F / \rho C \mu$, where $R$ is the reflectivity, $\rho$ is the density, $C$ is the specific heat and $\mu$ is the thermal diffusion length, and the following thermal expansion $H$ is expressed as $H=$ $\alpha \mu \Delta T=(1-R)(1-\eta) F \alpha / \rho C$, where $\alpha$ is the thermal expansion coefficient ${ }^{[11]}$. Inserting the following typical values of $R=$ $0.16^{[15]}, \eta=0.06^{[16]}, F=2.3 \times 10^{-3} \mathrm{~J} / \mathrm{cm}^{2}, \alpha=4.5 \times 10^{-6}$ $\mathrm{K}^{-1[17]}, \rho C=2.8 \mathrm{Jcm}^{-3} \mathrm{~K}^{-1}[17]$, the thermal expansion of a $\mathrm{ZnO}$ crystal is estimated as $H=2.9 \times 10^{-9} \mathrm{~cm}$. Though the thermal expansion is very small, the expansion is caused during the laser pulse duration of $\tau=5 \mathrm{~ns}$, resulting in the high average velocity $v=H / \tau=0.58 \mathrm{~cm} / \mathrm{s}$ and the high acceleration $a=H / \tau^{2}=1.2 \times 10^{8} \mathrm{~cm} / \mathrm{s}^{2}$. The huge acceleration being about hundred thousand times larger than the gravitational acceleration can cause the LIM over the adhesion forces such as an electrostatic force and an atomic force ${ }^{[11]}$.

\section{Atmospheric Alignment of a $\mathrm{ZnO}$ nanowire using Laser-induced Motions and Electrical Field}

O. Harnack et. al. have reported a dielectrophoretic alignment of $\mathrm{ZnO}$ nanorods with a typical size of $30 \mathrm{~nm}$ in diameter and $300 \mathrm{~nm}$ in length by the use of a solution including the nanorods and an electrical field applied by an alternating-current (AC) power supply at 1 to $10 \mathrm{kHz}{ }^{[7]}$. The electrical field causes a dipole moment due to a polarization of a $\mathrm{ZnO}$ nanorod, and the inhomogeneous electrical field induces a movement toward the highest field density. Similarly, it is considered that $\mathrm{ZnO}$ micro / nanocrystals can be aligned by the LIM and the inhomogeneous electrical field in the air. Therefore, we demonstrated the alignment method using trianglear $\mathrm{Al}$ electrodes evaporated on a silica glass substrate where a direct-current (DC) power supply was employed because any solution was not used in our method. Figure 5 shows serial images of the LIM controlled by the applied electrical field, and a schematic was also shown in the lower right side. From Fig. 5 (a) to (g), the $\mathrm{ZnO}$ nanowire was moved toward the electrode along the inhomogeneous electrical field by the LIM, and then, the nanowire was randomly moved away from the electrode after the applied DC power was turned off as shown in Fig. 5 (h) to (j).

It should be noted that the considerably long $\mathrm{ZnO}$ nanowire with several tens $\mu \mathrm{m}$ in length could be moved and aligned. In addition, this technique can be applied not only to $\mathrm{ZnO}$, but also to various materials with a high absorption coefficient for the UV laser light as well.

\section{Summary}

Lasing characteristics of a single $\mathrm{ZnO}$ microsheet were investigated by a UV laser light excitation with thirdharmonic of a Q-switched Nd:YAG laser beam $(\lambda=355 \mathrm{~nm}$, 
$\tau=5 \mathrm{~ns})$. According to the emission spectra observation from the microsheet, lasing spectra with a mode structure on broad emissions and a low lasing threshold of 49 $\mathrm{kW} / \mathrm{cm}^{2}$ could be observed. Since this threshold was as low as that of $\mathrm{ZnO}$ nanosheet reported in our previous study, $\mathrm{ZnO}$ micro / nanocrystals can be superior laser mediums as building blocks. In addition, laser-induced motions (LIM) of $\mathrm{ZnO}$ crystals were observed at high excitation intensities, and an alignment method of a $\mathrm{ZnO}$ nanowire was demonstrated using LIM and inhomogeneous electrical fields. As a result, an atmospheric alignment method of $\mathrm{ZnO}$ micro / nanocrystals could be realized by LIM and designed electrical fields. This technique can be applied to the fabrication of a UV laser diode using $\mathrm{ZnO}$ micro / nanocrystals as building blocks.

\section{Acknowledgments}

A part of this work was supported by a Grant-in-Aid for Scientific Research from the Japan Society for the Promotion of Science (JSPS, No. 20360142) and Special Coordination Funds for Promoting Science and Technology from Japan Science and Technology Agency is also acknowledged.

\section{References}

[1] Z. K. Tang, G. K. L Wong, P. Yu, M. Kawasaki, A. Ohtomo, H. Koinuma and Y. Segawa, Appl. Phys. Lett. 72, 3270 (1998).

[2] E. S. Jang, X. Chen, J. H. Won, J. H. Chung, D. J. Jang, Y. W. Kim and J. H. Choy Appl. Phys. Lett. 97, 043109 (2010).

[3] L. K. Vugt, S. Ruhle and D. Vanmaekelbergh, Nano Lett. 6, 2707 (2006).
[4] M. A. Zimmler, F. Capasso, S. Müller, and C. Ronning, Semicond. Sci. Technol. 25, 024001 (2010).

[5] K. Okazaki, D. Nakamura, M. Higashihata, P. Iyamperumal and T. Okada, Opt. Exp. 19, 20389, (2011).

[6] K. Okazaki, K. Kubo, T. Shimogaki, D. Nakamura, M. Higashihata and T. Okada, Adv. Mat. Lett. 2, 354 (2011).

[7] O. Harnack, C. Pacholski, H. Weller, A. Yasuda and J. M. Wessels, Nano Lett. 3, 1097 (2003).

[8] A. W. Maijenburg, M. G. Maas, E. J. B. Rodijk, W. Ahmed, E. S. Kooij, E. T. Carlen, D. H. A. Blank and J. E. ten Elshof, J. Colloid Interface Sci. 355, 486 (2011).

[9] J. H. Park and J. G. Park, Curr. Appl. Phys. 6, 1020 (2006).

[10] S. Adachi, Kluwer Academic Chap. D2 (1999).

[11] A. C. Tam, W. P. Leung, W. Zapka and W. Ziemlich, J. Appl. Phys. 71, 3515 (1992).

[12] T. W. Chiu, K. Tonooka, N. Kikuchi, Thin Solid Films 516, 5941 (2008).

[13] H. Pan, N. Misra, S. H. Ko, C. P. Grigoropoulos, N. Miller, E. E. Haller and O. Dubon, Appl. Phys. A 94, 111 (2009).

[14] S. A. Studenikin and Michael Cocivera, J. Appl. Phys. 91, 5060 (2002).

[15] D. G. Thomas, J. Phys. Chem. Solids 15, 86 (1960).

[16] S. F. Chichibu, T. Onuma, M. Kubota, and A. Uedono, J. Appl. Phys. 99, 093505 (2006).

[17] G. G. Gadzhiev, High Tmperature 41, 778 (2003).

(Received: June 07, 2011, Accepted: July 13, 2012) 